\title{
GA Based Digital Image Watermarking for Enhanced Robustness and Imperceptibility
}

\author{
R. Surya Prakasa Rao ${ }^{1}$, Dr. P. Rajesh Kumar ${ }^{2}$ \\ Dept., of ECE, AU College of Engineering, Visakhapatnam, India. \\ Dept., of ECE, AU College of Engineering, Visakhapatnam, India.
}

\begin{abstract}
The goal of image watermarking is to hide the specific information into host image and the presence of the watermark cannot be realized by human visual. The watermarking algorithm design should meet the criteria such as the rightful ownership protection, robustness against the image processing attacks and watermark imperceptibility. In this paper an optimal watermarking scheme based on singular value decomposition (SVD) and Discrete wavelet transform (DWT) using multi-objective genetic algorithm (GA) optimization is presented. The singular values of the watermark are embedded in a detail sub-band of host image after third level approximation. The GA algorithm is used to achieve the highest possible robustness and transparency. Experimental results gives improved performance in term of transparency and robustness after attacks by the proposed method compared to other existing methods.
\end{abstract}

Keywords: Digital Image watermarking, DWT, SVD, GA, Robust, Imperceptibility

\section{Introduction}

In the watermarking algorithm procedure, only the real owner can extract watermark correctly from a watermarked image. The watermarking proposed (1) and improved by the researchers in the SVD domain[7-22] in that the watermark is embedded into the singular value of the host image. In [7-15, 17, 19], the host image is transformed first (mostly using the wavelet transform) before performing the SVD. In SVD watermarking techniques $[7-15,17,19]$, the visual watermark is embedded into the singular value matrix of the host image in the transformed domain by selecting the suitable scaling factor to achieve the imperceptibility aspect in the watermarked image. The two approaches are used to embed the watermark information into a host image such as directly insert a watermark image into host image singular value matrix [1,15-22], and only inject the watermark singular value into the singular value [7-14] of host image. Both types of these methods gives a good quality watermarked image in terms of the PSNR, and robust against various attacks in terms of the correlation coefficient [1,7-22]. Still, the image quality is degraded and the transparency of the watermark is improved by a lower value of scaling factor but it is less robust against various geometric attacks. The tradeoff between these two has been addressed to get the suitable scaling factor determination as an optimization problem [16,18,20]. The genetic algorithm [16],the tiny genetic algorithm [18] and differential evolution algorithm [20] is used to get the optimum scaling factor to achieve the robustness and to improve the image quality of watermarked image. However, a major flaw is found in the SVD watermarking as reported in [2-6]. GA to optimize the watermark strength as used by Vahedi et al.[24].Basically RGB, YCbCr, YIQ, HIS, HSV and other color space models which are used by the researchers for the host image in their digital watermarking methods. The above mentioned color models are compared i.e. change in one component may affect the other components of image and image components are not independent. The compared color host image to use only one color component at a time for embedding the watermark data which forces the constraints used by the researches. however, there exist some un compared color models such UCS, etc.[25],[26], which may be used in color image watermarking to increase the durability and resolution by using all the color image components of host images, Saraswat and Arya [26] used UCS for color transfer of images and detected that UCS outperforms the other un corresponded color spaces. In the proposed scheme is combining DWT and SVD with genetic algorithm to find the optimal scaling factor and embed the singular value of watermark in singular value of $3^{\text {rd }}$-level DWT approximation matrix of original image.

The proposed image watermarking based on Genetic algorithm is presented in the remaining part as follows: section II describes the details of related work. The complete detail of proposed watermarking scheme is illustrated in section III. Section IV reveals the details of simulation obtained after the implementation of proposed approach. Finally the conclusions are described in section V. 


\section{A. Discrete Wavelet Transform}

\section{Related Work}

Discrete Wavelet Transformation is a wavelet filter used to transform the image. The Haar and Daubchies are commonly used filters. By using these filters single level decomposition gives four frequency components named as HH1, HL1, LH1, and LL1. In second level decomposition, apply transform to LL1 and so on to get the desired one. The model of three- level decomposition of image [21] as shown in fig.1.

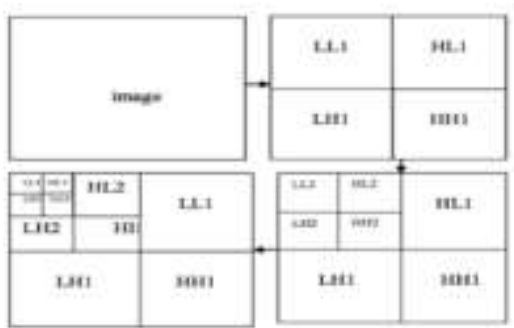

Fig.1: Three LevelDWT

Discrete Wavelet Transformation is very suitable to identify the areas in the cover image where a secret image can be embedded effectively. This property allows the exploitation of the masking effect of the human visual system such that if a DWT co-efficient is modified, it modifies only the region corresponding to that coefficient. The embedding watermark in the lower frequency sub-bands may degrade the image as generally most of the Image energy is stored in these sub-bands. However it is more robust. The high frequency part contains information about the edge of the image so this frequency sub-bands are usually used for watermarking since the human eye is less sensitive to changes in edges. The DWT splits the signal into high and low frequency parts. The low frequency part contains coarse information of signal while high frequency part contains information about the edge components. The high frequency components are usually used for watermarking since the human eye is less sensitive to changes in edges.

\section{B. Singular Value Decomposition}

SVD is a mathematical tool to analyze matrices. In SVD real matrix $I_{G}$ decomposed in to three matrices $I_{G}=X_{L} S Y_{R}{ }^{T}$ Where $X_{L}$ and $Y_{R}$ are orthogonal matrices in which columns of $X_{L}$ are left singular vectors and columns of $Y_{R}$ are right singular vectors of image $I_{G}$. $D$ is a diagonal matrix of singular values in decreasing order. The Singular values of grey scale original image are modified to embed the watermark image by applying multiple scaling factors, after that using GA modifications are made to get highest possible transparency and Robustness under certain attacks [10]. It is a tool in linear algebra, which is widely applied in many research fields such as principal component analysis, canonical correlation analysis and data compression. The component $\mathrm{S}$ is a singular value matrix in SVD domain, and is a diagonal matrix with nonnegative real numbers,

$S_{M N}=\left[\begin{array}{ccccc}S(1,1) & 0 & \ldots & 0 \\ 0 & \ddots & \ldots & 0 \\ \vdots & \vdots & \ddots & & \vdots \\ 0 & 0 & \ldots & & S(M, N)\end{array}\right]$

Where $S(1,1) \geq S(2,2) \geq S(3,3) \ldots \geq S(M, N) \geq 0$.

\section{Genetic Algorithm}

Genetic algorithm [22] is one of the mostly used optimization technique belonging to area of evolutionary computation. GA is mainly composed of three operations: selection, genetic operation and replacement. Initially, a population is randomly generated. The fitness function then uses objective values from objective function to evaluate the fitness of each chromosome. The fitter chromosome has the greater chance to survive during the revolution process.

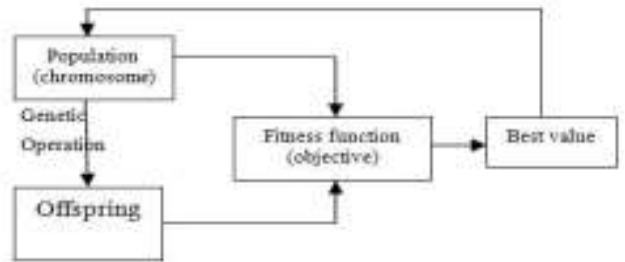

Fig.2: GA Cycle 
The objective function is the problem specific; its objective value can represent the system performance index (e.g., an error). Next, a particular group of chromosome is chosen from the population to be parents. The offspring is then generated from these parents by using genetic operation, which normally are crossover and mutation, similar to their parents, the fitness of the offspring is evaluated and used in replacement process in order to replace the chromosomes in the current population by the selected offspring. The GA cycle is the repeated until a desired termination criterion is satisfied, for example, if the maximum number of generations is reached or the objective value is bellowed the threshold.

\section{Proposed Approach}

In this proposal, The GA is used to obtain optimum scaling factors to meet up high Robustness under certain attacks. The Block Diagram of proposed approach as shown in Fig.3 and The steps needed for Embedding and Extraction is as follows:

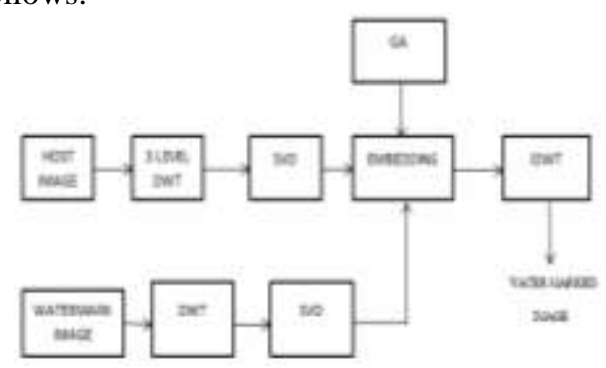

(a)

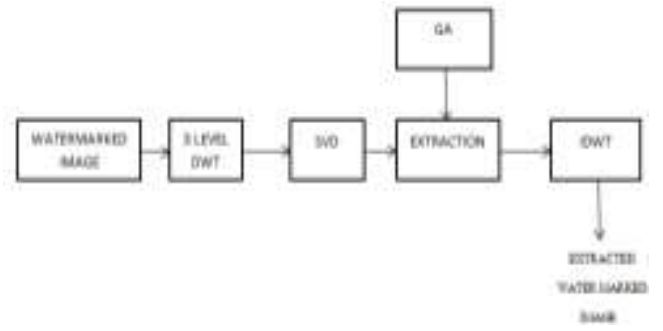

(b)

Fig.3: Block Diagram of ProposedWatermarking Approach (a) Embedding process (b) Extraction Process

\section{A. Embedding Procedure}

(i) Initialize the parameters of Genetic Algorithm: Select value for scaling factor SF from 0 to 1 and set the population size and the number of iterations.

(ii) Generate the 1st generation of individuals randomly. Perform the watermarking embedding procedure, for each iteration different watermarked image is produced.

(iii) Decompose the image $I_{G}$ in to four sub bands using three levels Haar DWT.

(iv) Apply SVD to $L L_{3}$ sub band and also for remaining bands, as $I_{G i}=X_{3 i} D_{3 i} Y_{3 i}^{T}$, Where $i=L L, L H, H L, H H$.

(v) Decompose the watermark image $\mathrm{I}_{\mathrm{W}}$ in to four sub bands using one level Haar DWT.

(vi) Apply SVD to LL sub band and also for remaining bands as $I_{w i}=X_{w i} D_{w i} Y_{w i}^{T}$, Where $i=L L, L H, H L, H H$.

(vii) Modify the singular value of $\mathrm{I}_{\mathrm{G}}$ by embedding singular value of $\mathrm{W}_{\mathrm{G}}$ in $\mathrm{LL}$ sub band and for remaining such that

$$
D_{3 m i}=D_{3 i}+S_{i} * D_{w i} \text {, where } i=L L, L H, H L, H H \text {. }
$$

Where $D_{3 m i}$ is the modified singular values and $\mathrm{S}$ is the scaling factors.

(viii) Apply inverse SVD on the altered singular values of all bands. The new bands are denoted as $I_{G W L L}, I_{G W L H}$, $I_{G W H L}, I_{G W H H}$.

(ix) To obtain the watermarked image $\left(I_{w}\right)$ applying three level inverse DWT using modified coefficients $I_{G W}$.

(x) Apply different attacks on the watermarked image.

\section{B. Extraction Procedure}

It is applied to extract the watermark from each attacked watermarked image.

(i) Apply three level Haar DWT to decompose the watermarked image into four sub bands.

(ii) Apply SVD to $L L_{3 w}$ sub band and also for remaining bands, as $I_{G w i}=X_{3 w i} D_{3 w i} Y_{3 w i}^{T}$, Where $i=L L, L H, H L, H H$.

(iii) Compute $D_{3 w E i}=\left(D_{3 w i}-\bar{D}_{3 i}\right) / S_{i}$ where $i=L L, L H, H L, H H$. Here the $D_{3 w E i}$ is the singular matrix of the respective $i_{t h}$ band of extracted watermark. 
(iv) Distorted bands will be obtained by performing SVD on the obtained singular values of all bands as $I_{G W L L}{ }^{*}$, $I_{G W L H}{ }^{*}, I_{G W H L}{ }^{*}, I_{G W H H}{ }^{*}$.

(v) Then the final watermark can be extracted byapplying inverse DWT on the obtained distorted wavelet bands as $I_{W}{ }^{*}$.

(vi) Compute the robustness and imperceptibility [22] using equations (2) and (3) as

$$
\begin{aligned}
& N C=\sum_{i=1}^{M} \sum_{j=1}^{N} \frac{w(i, j) * w^{*}(i, j)}{(w(i, j))^{2} * w^{*}((i, j))^{2}}(2) \\
& P S N R=10 * \log \left(255^{2} /{ }_{M S E}\right)
\end{aligned}
$$

Where

Where

$$
M S E=\frac{1}{M X N} \sum_{i=1}^{M} \sum_{j=1}^{N}\left(I(i, j)-I^{*}(i, j)\right)^{2}(4)
$$

$I=$ original image

$I^{*}=$ watermarked image

(vii) The Fitness Function $(\mathrm{FF})$ is evaluated as $\mathrm{FF}=\mathrm{PSNR}+100 * \mathrm{NC}$.

(viii) Select the best fitness values based on theRobustness and Imperceptibility.

(ix) Generate the new population performing the crossover and mutation on the selected population.

(x) Repeat the steps from 2 to 9 until the result has been reached.

\section{Simulation Results}

The proposed approach implemented using Matlab R2013a taking original image of size 256x256and watermark image of size 64x64. The proposed DWT-SVD-GA based watermarking algorithm is accomplished through genetic algorithm taking population size of 10 . The entire algorithm is repeated for overall population and based on the obtained results one values is determined as optimal values at which the proposed approach obtained the optimal results. The test images considered for the implementation is shown below.

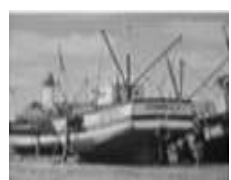

(a)

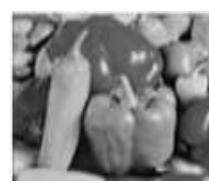

(b)

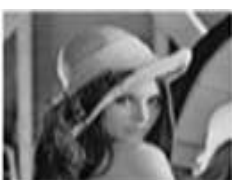

(c)

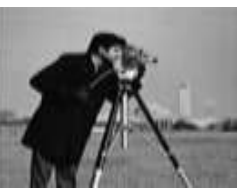

(d)

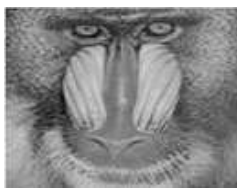

(e)

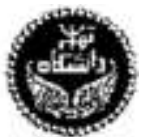

(f)

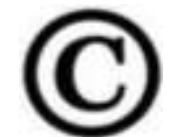

(g)

Fig.4:Test Images (a)-(e) Host Images and (f)-(g) Watermark Images, (a) Boat (b) Peppers (c) Lena (d) Cameraman (e) Mandrill (f) Binary watermark (g) Logo Watermark

Initially the watermarked image is not subjected to any attack. The watermark is extracted directly from the watermarked image through the extraction process specified in third section and the obtained results are shown below.
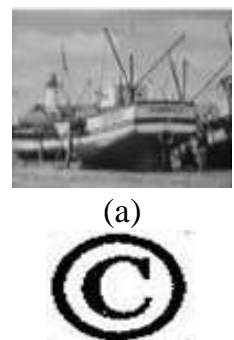

(f)
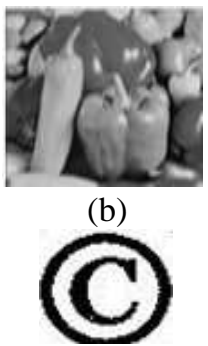

(g)
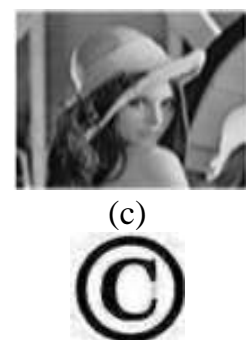

(h)
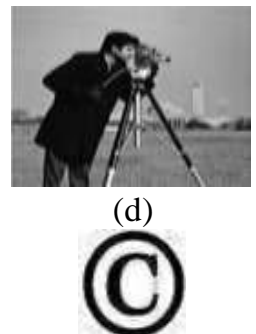

(i)

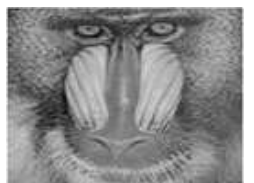

(e)

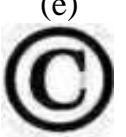

(j)

Fig.5: Results Observed (a)-(e) Watermarked Images (f)-(j)Extracted Watermark images 


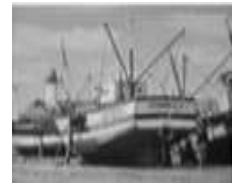

(a)

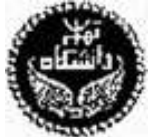

(f)

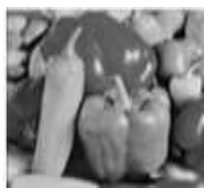

(b)

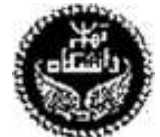

(g)

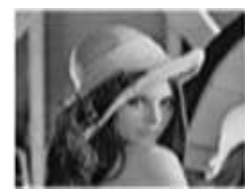

(c)

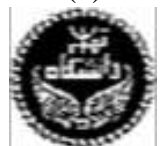

(h)

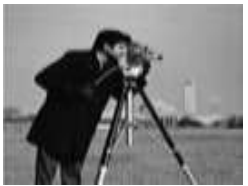

(d)

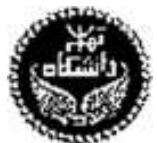

(i)

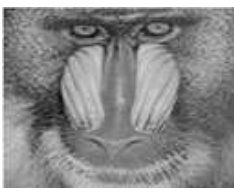

(e)

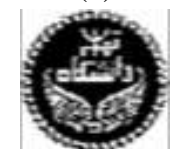

(j)

Fig.6: Results Observed (a)-(e) Watermarked Imagesand (f)-(j)Extracted Watermark images

Then the extracted watermark images are processed for analysis by measuring the NC, PSNR and MSE. The mathematical representations are shown in Equations (2), (3) and (4). The obtained PSNR, MSE and NC for the above test results (Figure.6) are described in table.1.

Table.1:Comparison between proposed and conventional approaches under no attack scenario

\begin{tabular}{|l|c|c|c|c|c|c|}
\hline \multirow{2}{*}{ Image } & \multicolumn{3}{|c|}{ DWT-GA (Vahedi et.al) [24] } & \multicolumn{3}{c|}{ DWT-SVD-GA (Proposed) } \\
\cline { 2 - 7 } & MSE & PSNR & NC & MSE & PSNR & NC \\
\hline Lena & 4.3531 & 41.7428 & 0.9730 & 2.9414 & 43.4452 & 0.9793 \\
\hline Pepper & 7.6888 & 39.2722 & 0.9758 & 3.8097 & 42.3219 & 0.9777 \\
\hline Boat & 5.9899 & 40.3356 & 0.9442 & 3.0704 & 43.2589 & 0.9489 \\
\hline Cameraman & 5.3388 & 40.8564 & 0.9523 & 2.3298 & 44.4576 & 0.9571 \\
\hline Mandrill & 7.6022 & 39.3124 & 0.9412 & 2.8661 & 43.5579 & 0.9463 \\
\hline
\end{tabular}

Table1 shows the comparison between the existing method proposed by Vahedi et. al [24] and the proposed method under no attack scenario. From table.1, it can be observed that the proposed approach obtained an increased PSNR and NC values compared to the conventional approach for all test images. Figure.7 and figure. 8 illustrates the details of the obtained PSNR and NC values for both conventional and proposed approaches for all test images.

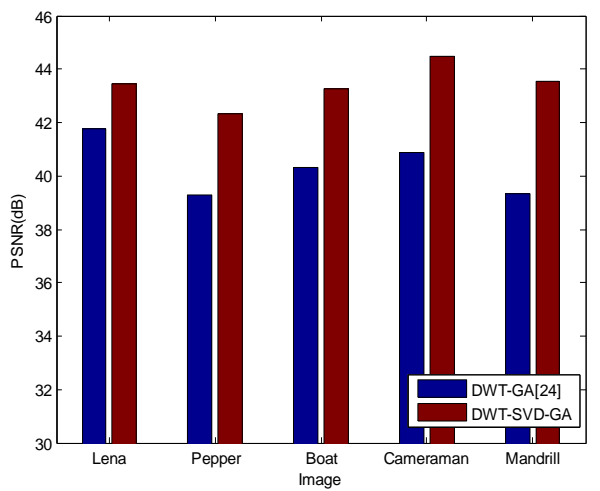

Fig.7: PSNR observations for all test images

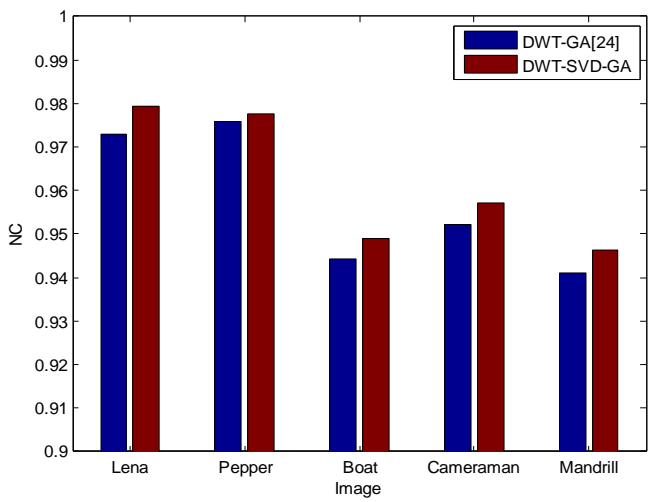

Fig.8: NC observations for all test images 
Further the performance of proposed approach is analyzed through various attacks scenarios. Under this evaluation the test image is subjected to various types if attacks and then the proposed approach is applied over the attacked watermarked image to extract the watermark. Here the attacks considered are image processing attacks such as Gaussian noise attack, salt \& pepper noise, Poisson noise, Wiener filtering, median filter with varying size $(3 * 3$ and $5 * 5)$, mean filtering with varying size $(3 * 3$ and $5 * 5)$ and geometric attacks such as crop with varying $\%$ of crop, histogram equalization, rotation and scaling with varying scaling factor $(0.8,1.2$ and 1.5). Then the numerical metrics such as PSNR, MSE and NC are evaluated for the extracted watermark. The obtained rests of Lena image for image processing attacks and the geometric attacks are tabulated in table. 2 and table. 3 respectively.

Table.2: Comparative analysis between proposed and conventional approaches for image processing attacks

\begin{tabular}{|c|c|c|c|c|c|c|}
\hline \multirow{2}{*}{ Attack } & \multicolumn{2}{|c|}{ DWT-GA (Vahedi et.al) [24] } & \multicolumn{2}{c|}{ DWT-SVD-GA (Proposed) } \\
\cline { 2 - 6 } & MSE & PSNR & NC & MSE & PSNR & NC \\
\hline Gaussian & 9.7897 & 38.2231 & 0.9204 & 6.3139 & 40.1278 & 0.9258 \\
\hline Salt \& pepper & 11.6740 & 37.4586 & 0.9149 & 7.7507 & 39.2374 & 0.9186 \\
\hline Poission & 9.7800 & 38.2274 & 0.9562 & 6.2894 & 40.1447 & 0.9597 \\
\hline Wiener & 5.8704 & 40.4441 & 0.9726 & 4.4333 & 41.6635 & 0.9733 \\
\hline Medin Filter (3*3) & 7.5798 & 39.3342 & 0.9785 & 4.3198 & 41.7762 & 0.9789 \\
\hline Median filter (5*5) & 18.6982 & 35.4128 & 0.9029 & 12.008 & 37.3387 & 0.9048 \\
\hline Mean Filter (3*3) & 20.4536 & 35.0231 & 0.6577 & 13.0128 & 36.9871 & 0.6779 \\
\hline Mean Filter (5*5) & 37.9499 & 32.3387 & 0.6577 & 23.3776 & 34.4428 & 0.6762 \\
\hline
\end{tabular}

Table.3: Comparative analysis between proposed and conventional approaches for Geometric attacks

\begin{tabular}{|c|c|c|c|c|c|c|}
\hline \multirow{2}{*}{ Attack } & \multicolumn{3}{|c|}{ DWT-GA (Vahedi et.al) [24] } & \multicolumn{2}{c|}{ DWT-SVD-GA (Proposed) } \\
\cline { 2 - 7 } & MSE & PSNR & NC & MSE & PSNR & NC \\
\hline Crop (10\%) & 19.4716 & 35.2310 & 0.9378 & 11.7012 & 37.4485 & 0.9441 \\
\hline Crop (20\%) & 34.2500 & 32.7842 & 0.8861 & 27.2189 & 33.7821 & 0.8902 \\
\hline Crop (40\%) & 86.2066 & 28.7754 & 0.7803 & 56.9955 & 30.5724 & 0.7885 \\
\hline HEQ & 13.7563 & 36.7458 & 0.9503 & 10.8588 & 37.7742 & 0.9556 \\
\hline Scale*0.8 & 10.5519 & 37.8975 & 0.9478 & 8.4011 & 38.8859 & 0.9509 \\
\hline Scale*1.2 & 11.7039 & 36.4475 & 0.9388 & 10.3350 & 37.9877 & 0.9401 \\
\hline Scale*1.5 & 25.1229 & 34.1289 & 0.9406 & 16.2469 & 36.0231 & 0.9463 \\
\hline Rotation & 14.3830 & 36.5523 & 0.8757 & 13.0173 & 36.9856 & 0.8842 \\
\hline
\end{tabular}

From table.2, it can be observed that the proposed method achievesbetter robustness for all types of attacks mentioned in this paper.The obtained PSNR for all the cases reveals that the proposed approach obtained an enhanced image quality. Thus the proposed approach is more robust and also gives more imperceptibly Compared to the conventional approach. Figure.9 and figure.10 describes the details of PSNR and NC respectively. Figure.9 determines the obtained PSNR under both image processing and geometric attacks of conventional and proposed approaches, similarly figure.10 gives NC details.

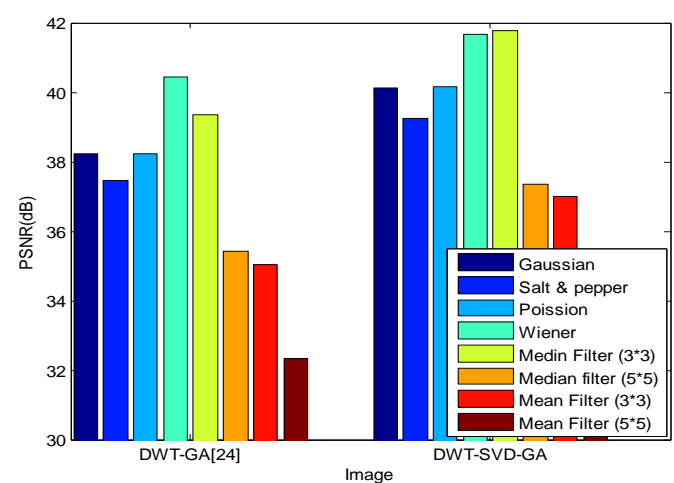

(a)

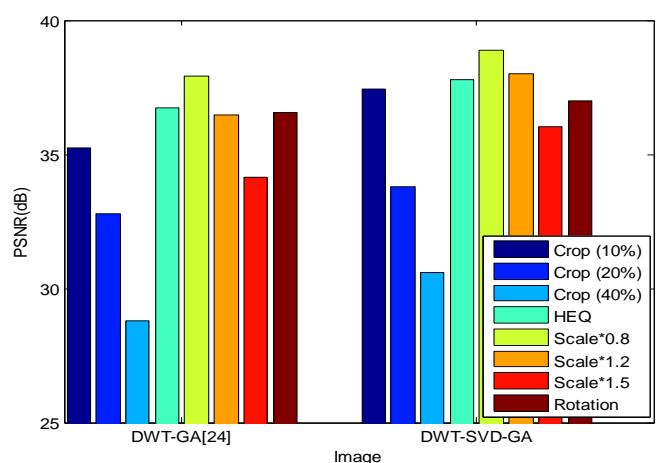

(b)

Fig.9: PSNR observations for various types of attacks (a) Image processing attacks (b)Geometric Attacks 


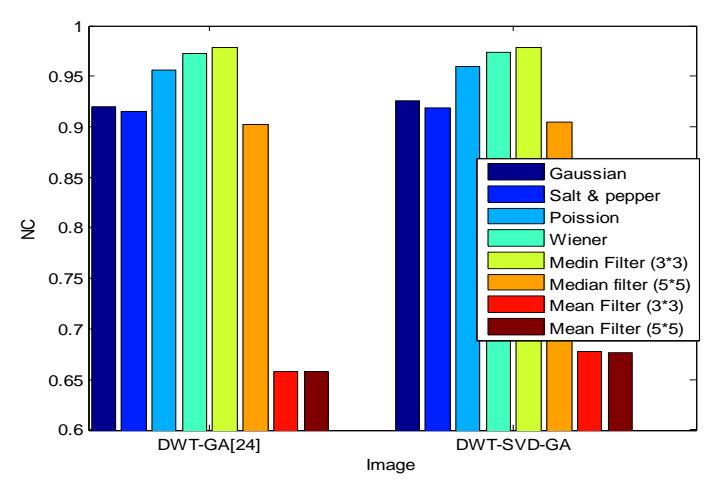

(a)

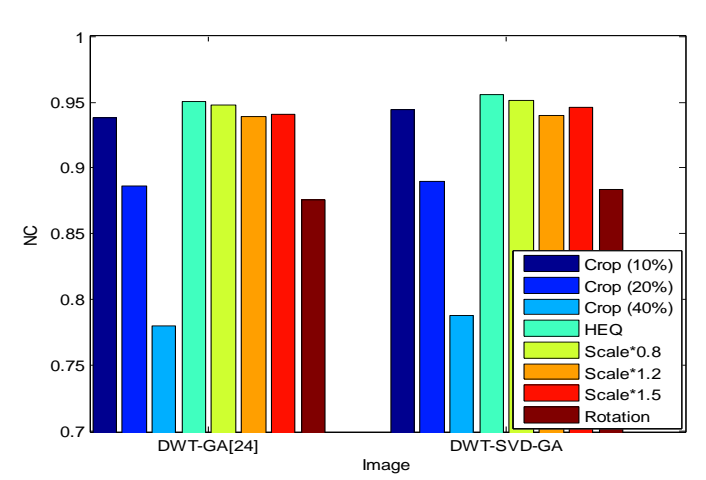

(b)

Fig.10: NC observations for various types of attacks (a) Image processing attacks (b)Geometric Attacks

Form the above figures it can be observed that the geometric attacks increases the distortion in the extracted watermark image compared to image processing attacks. The very less PSNR and NC values are observed at cropping attack (40\%). In that case also the PSNR and NC of proposed approach is observed to be high. Thus the proposed approach is more robust for both image processing and geometric attacks compared with conventional approaches.

\section{Conclusion}

In this simulation various tests are done on different cover images named as Lena, boat, Pepper and mandrill with size 256 x 256, where the enhanced PSNR and NC values are observed between original watermark and extracted watermark after applying attacks like Gaussian noise, salt and pepper, wiener filter, Poisson noise, cropping, median filtering, mean filtering, histogram equalization, scaling and rotation compared to conventional approach. Genetic Algorithm used to find out Superior scaling factor for watermark embedding. The above results proved that Performance of proposed watermarking scheme is good in terms of Robustness and Imperceptibility for different attacks.

\section{References}

[1]. Liu RZ, Tan. TN. An SVD-based watermarking scheme forProtecting rightfulownership. IEEE Trans Multimedia 2002;4(1):121-8.

[2]. Zhang XP, Li. K. Comments on an SVD-based watermarking scheme for protec-ting rightful ownership. IEEE Trans Multimedia 2005;7(3):593-4.

[3]. Rykaczewski R. Comments on SVD-based watermarking scheme for protectingrightful ownership. IEEE Trans Multimedia 2007;9(2):421-3.

[4]. Changzhen X, Fenhong G, Zhengxi L. Weakness analysis of singular valuebased watermarking. In: Int. Conf. Mechantronics and Automation.2009. p.2596-601.

[5]. Changzhen X, Ward RK, Xu J. On the security of singular value based water-marking. In: IEEE Int. Conf. Image Proc. 2008. p. 437-40.

[6]. Sadek RA. Blind synthesis attack on SVD based watermarking techniques. In:Int. Conf. Computational Intelligence for Modeling Control and Automation.2008. p. 140-5

[7]. Rastegar S, Namazi F, Yaghmaie K, Aliabadian A. Hybrid Watermarking based onsingular value decomposition and Radon transform. AEU-Int J Electron Com-mun 2011;65(7):658-63

Ganic E, Eskicioglu AM. Robust embedding of visual watermarks using DWT-SVD. J Electron Imaging 2005;14(4):043004.

[8]. Bhatnagar G. A new facet in robust digital watermarking framework. AEU-Int JElectron Commun 2012;66(4):275-85.

[9]. Bhatnagar G, Wu QMJ. Biometrics inspired watermarking based on a frac-tional dual tree complex wavelet transform. Future Gener Comput Syst2013;29(1):182-95.

[10]. Song C, Sudirman S, Merabti M. A robust region-adaptive dual Image water-marking technique. J Vis Commun Image Represent 2012;23(3):549-68.

[11]. Bhatnagar G, Wu QMJ, Raman B. A new robust adjustable Logo watermarkingscheme. Computer Security 2012;31(1):40- 58

[12]. Bhatnagar G, Wu QMJ, Raman B. A new logo watermarking basedon redundant fractional wavelet transform. Math Comput Model 2012,http://dx.doi.org/10.1016/j.mcm.2012.06.002.

[13]. Lagzian S, Soryani M, Fathy M. Robust watermarking scheme based in RDWT-SVD: embedding data in all subbands. In: Int.Symp. Artificial Intelligence andSignal Processing. 2011. p. 48-52.

[14]. Makbol NM, Khoo. BE. Robust blind image watermarkingScheme based onredundant discrete wavelet transform and singular value decomposition. AEU-Int J Electron Commun, 2013;67(2):102-12.

[15]. Aslantas V. A singular-value decomposition-based image Watermarking usinggenetic algorithm. AEU-Int J Electron Commun 2008;62(5):386-94.

[16]. Faragallah OS. Efficient video watermarking based on singularvalue decompo-sition in the discrete wavelet transform domain. AEU-Int J Electron Commun2013;67(3):189-96.

[17]. Lai. CC. A digital watermarking scheme based on singular Value Decomposition and tiny genetic algorithm. Digit Signal Process 2011;21(4):522-7.

[18]. Lai CC, Tsai. CC. Digital image watermarking using discrete wavelettransform and singular value decomposition. IEEE Trans Instrum Meas2010;59(11):3060-3. 
[19]. Aslantas V. An optimal robust digital image watermarking based on SVD usingdifferential evolution algorithm. Opt

Commun 2009;282(5):769-77.

[20]. "Digital Image Watermarking Using 3 level Discrete Wavelet transform" Pratibha Sharma and Shanti Swami Conference on Advances in Communication and Control Systems2013.

[21]. Ali A-Haj and Aymen Abn-Errub, Performance Optimization of Discrete Wavelets Transform Based Image WatermarkingUsing Genetic Algorithm", Journal of Computer Science 4(10):834-841,2008.

[22]. A. A.Mohammad, A. Alhaj, and S. Shaltaf, "An improved SVDbased watermarking scheme for protecting rightfulownership," Signal Processing, vol. 88, no. 9, (2008), pp. 2158-2180.

[23]. E. Vahedi, R. A. Zoroofi, M. Shiva, Toward a new wavelet- based watermarking approach for color images using bio- inspired optimization principles, Digital Signal Processing,Elsevier, 22, 153- 162, 2012.

[24]. C.-H. Chou, K.-C. Liu, A perceptually tuned watermarking scheme for color images, IEEE Transactions on Image Processing, 19, 2966-2982, 2010

[25]. M. Saraswat, K. Arya, Colour normalization of histopathological images, Computer Methods in Biomechanics and Biomedical Engineering: Imaging \& Visualization, Taylor \& Francis, 1-13, 2013. 